\title{
Radiofrequency plasma antenna generated by femtosecond laser filaments in air
}

\author{
Y. Brelet ${ }^{1}$, A. Houard ${ }^{1}$, G. Point ${ }^{1}$, B. Prade ${ }^{1}$, L. Arantchouk ${ }^{2}$, J. Carbonnel $^{1}$, Y.-B. \\ André $^{1}$, M. Pellet ${ }^{3}$ and A. Mysyrowicz ${ }^{1}$ \\ ${ }^{1}$ Laboratoire d'Optique Appliquée, ENSTA ParisTech, Ecole Polytechnique, CNRS, 91761, \\ Palaiseau, France \\ ${ }^{2}$ Laboratoire de Physique des Plasmas, Ecole Polytechnique, CNRS, Palaiseau, France \\ ${ }^{3}$ Etat-major de la Marine Nationale, France
}

We demonstrate tunable radiofrequency emission from a meter-long linear plasma column produced in air at atmospheric pressure. A short-lived plasma column is initially produced by femtosecond filamentation and subsequently converted into a long-lived discharge column by application of an external high voltage field. Radiofrequency excitation is fed to the plasma by induction and detected remotely as electromagnetic radiation by a classical antenna.

In a plasma antenna the metallic tube is replaced by a plasma column ${ }^{1}$. Advantages of plasma antennas include fast turn-on and turn-off times, stealth features (a low radar cross-section when deenergized) and reconfigurable shape. This concept has been demonstrated with low density plasma generated inside dielectric tubes. The tube is filled with a low-pressure noble gas which is ignited by applying an intense electric field ${ }^{2-5}$. Radiation performances of such plasma antenna have been compared favorably with those of conventional metal antenna ${ }^{2-6}$. They are particularly well-suited for digital communication of data streams ${ }^{7}$. The suggestion that plasma produced by laser pulses can be employed to transport microwave radiation was published more than four decades ago ${ }^{8}$. A pioneer work demonstrated the feasibility of radiofrequency (RF) plasma using a nanosecond laser to induce a discharge in air ${ }^{1}$. More recently attention has been paid to the potentiality of femtosecond filamentation for guiding microwave radiation under atmospheric conditions ${ }^{9-12}$. During filamentation ${ }^{13-15}$ a weakly ionized plasma column is created in the wake of an intense fs laser pulse, with an initial electron density of $\sim 10^{16} \mathrm{~cm}^{-3}, \mathrm{a} \sim 100 \mu \mathrm{m}$ diameter and a nanosecond recombination time $^{16}$. The region over which plasma is produced is much longer than the classical Rayleigh length, reaching hundreds of meters in the best cases. Unfortunately, the rapid decay of the filament plasma is a major drawback for the realization of a RF antenna.

In this paper we report the demonstration of a tunable RF plasma antenna based on femtosecond filamentation operating in air at normal pressure. A meter-long plasma column is first initiated by filamentation, and then converted into a long-lived straight discharge ${ }^{17-23}$ by application of a high voltage (HV). Tunable RF oscillation coupled by induction to the plasma is radiated and detected at a distance with a classical reception patch antenna.

The experimental setup is illustrated in Fig. 1. The Ti:Sa CPA laser source used in the experiments (Enstamobile) delivers laser pulses at a repetition rate of $10 \mathrm{~Hz}$, with an energy $\mathrm{E}=300 \mathrm{~mJ}$, a pulse duration $\mathrm{t}_{\mathrm{p}} \approx 50 \mathrm{fs}$, and a central wavelength $\lambda_{0}=800 \mathrm{~nm}$. The laser pulse was chirped to a duration of $700 \mathrm{fs}$ corresponding to a laser peak power of $\sim 0.5 \mathrm{TW}$ in order to reduce the risk of damage on optical elements and mirrors. The $4 \mathrm{~cm}$ diameter femtosecond laser beam was focused by a $\mathrm{f}=5 \mathrm{~m}$ lens, producing a bundle of $\sim 50$ ionizing filaments with a diameter of 2-3 $\mathrm{mm}$ extending over two meters around the geometrical focus of the lens. The plasma column formed by this bundle connected two metallic electrodes separated by a distance D. A high voltage was applied to one of the electrode while the other was grounded. A first series of experiments to test the viability of the concept were performed on $5 \mathrm{~cm}$ long discharges by applying a DC voltage of $40 \mathrm{kV}$. Meter long discharges were obtained by applying HV bursts from a compact Tesla coil ${ }^{23}$. This homemade HV generator was synchronized with the laser pulses and delivered AC voltage with maximum amplitude of $\sim 365 \mathrm{kV}$. The length of the obtained discharges was comprised between 50 and $170 \mathrm{~cm}$. Most experiments were performed with a $96 \mathrm{~cm}$ long discharge (see Fig. 2).

An electric current reaching hundreds of Amperes with a 100 ns duration (FWHM) was measured between the two electrodes, proving that plasma lifetime has gained at least 2 orders of magnitude compared with filament in absence of HV discharge. Moreover, the electrical conductivity of the 
plasma is increased by at least three orders of magnitude going from $\sim 70$ Siemens $/ \mathrm{m}$ for the initial laser filament ${ }^{16}$ to $>4.8 \times 10^{4}$ Siemens $/ \mathrm{m}$ for the heated filament.

$\mathrm{RF}$ excitation was injected in the discharge plasma through an inductive coupler placed near the grounded electrode. Three different couplers were tested, with comparable performances, a solenoid, a Rogowski coil and a resonant cavity. The couplers were designed so as to offer a $50 \Omega$ coil impedance ${ }^{24}$. They were shielded and carefully insulated to avoid feedback currents towards the radiofrequency chain. A RF frequency wave with maximum power of $35 \mathrm{~W}$ was injected in the plasma column via the coupler. It was tunable between $100 \mathrm{MHz}$ and $1 \mathrm{GHz}$. From the measured incident and reflected powers, we deduced that at least $50 \%$ of the RF power was transmitted to the plasma. The RF signal emitted by the excited plasma column was collected by a planar antenna placed at a distance of $1.5 \mathrm{~m}$ from the plasma. The antenna detection band ranged from $100 \mathrm{MHz}$ to $11 \mathrm{GHz}$. The signal captured by the antenna was fed to an oscilloscope with a 2 Gsamples/sec sampling rate. The signal was treated by Fast Fourier Transform (FFT) in order to display the RF spectrum in the range of interest.

Figure 3 displays results obtained with the $5 \mathrm{~cm}$ long antenna. The figures show successively the spectral response of the reception antenna obtained with the RF alone (Fig. 3-a), with the discharge alone (Fig. 3-b), and with the RF excitation supplied to the discharge (Fig. 3-c). The injected excitation frequency at $970 \mathrm{MHz}$ is clearly apparent in the signal measured by the reception antenna (Fig 3-c). A comparison with the signal detected when the plasma discharge was replaced by a metallic copper wire of $5 \mathrm{~mm}$ radius is shown in Fig. 3-d. It shows that the plasma performance as a $\mathrm{RF}$ emitter is comparable, within a factor 4 , to a conventional antenna.

A typical result obtained with the meter-long plasma is shown in Fig. 4. The light curve (red online) shows the power spectral density which corresponds to the electric field detected by the reception antenna when a RF signal at $140 \mathrm{MHz}$ is injected in the plasma column with the solenoid coupler. A spectral line corresponding to the RF excitation fed to the coupler is clearly emerging from noise. The power spectral density of the background noise measured without discharge but with RF feeding is presented in black for comparison. The signal was comparable, within a factor 4 to that emitted by a copper wire of the same length as the discharge. We have obtained similar results when tuning the RF frequency between $100 \mathrm{MHz}$ and $1 \mathrm{GHz}$, corresponding to the range of the RF source. In future investigations, one can expect an improvement of the plasma antenna efficiency by increasing the length and the lifetime of the plasma.

We have demonstrated that it is possible to obtain a RF plasma antenna operating with air at normal pressure as a supporting medium. The plasma antenna is able to emit RF waves over a bandwidth spanning at least between $100 \mathrm{MHz}$ and $1 \mathrm{GHz}$ with an electrically controllable length.

\section{Acknowledgements}

This work was supported by the French Direction Générale de l'Armement (grant n 200795 091). The authors would like to thank Dr. Y. Liu, Dr. B. Forestier and A. Dos Santos for very fruitful discussions, and LEAT Nice who provided the slot antenna.

\section{References}

${ }^{1}$ T. J. Dwyer, J. R. Greig, D. P. Murphy, J. M Perin, R. E. Pechacek and M. Raleigh, IEEE Trans. Ant. Propa. AP-32, 2 (1984).

${ }^{2}$ G. Cerri, R. D. Leo, V. M. Primiani and P. Russo, IEEE Trans. Instrum. Meas. 57, 2 (2008).

${ }^{3}$ J. P. Rayner, A. P. Whichello and A. D. Cheetham, IEEE Trans. Plasm. Sci. 32, 1 (2004).

${ }^{4}$ G. G. Borg, J. H. Harris, D. G. Milijak and N. Martin, Appl. Phys. Lett. 74, 22 (1999).

${ }^{5}$ I. Alexeff, T. Anderson, S. Parameswaran, E. P. Pradeep, J. Hulloli and P. Hulloli, IEEE Trans. Plasm. Sci., 34, 2 (2006).

6 E. N.Istomin, D. M. Karfidov, I. M. Minaev, A. A. Rukhadze, V. P. Tarakanov, K. F. Sergeichev, A. Yu. Trefilov, Plasm. Phys. Rep. 32, 5 (2006).

${ }^{7}$ S. Haykin, Communication system, $4^{\text {th }}$ edition, John Wiley \& Sons, Inc. (2001).

${ }^{8}$ G. A. Askaryan, Zh. Eksp. Teor. Fiz. 55, 1400 (1968).

${ }^{9}$ S. B. Bodrov, D. I. Kulagin, Y. A. Malkov, A. A. Murzanev, A. I. Smirnov and A. N. Stepanov, J. Phys. D: Appl. Phys. 45, (2012).

${ }^{10}$ H. Nowakowska, Z. Zakrzewski and M. J. Moisan, J. Phys. D: Appl. Phys. 34, 1474 (2001). 
${ }^{11}$ V. D. Zvorykin A. O. Levchenko, A. G. Molchanov, I. V. Smetanin and N. N. Ustinovskii, Bulletin of the Lebedev Physics Institute, 37, 2 (2010).

${ }^{12}$ M. Chateauneuf, S. Payeur, J. Dubois and J. C. Kieffer, Appl. Phys. Lett. 92, 091104 (2008).

${ }^{13}$ A. Braun, G. Korn, X. Liu, D. Du, J. Squier, and G. Mourou, Opt. Lett. 20, 73 (1995).

${ }^{14}$ S L Chin, S A Hosseini, W Liu, Q Luo, F Théberge, N Aközbek, A Becker, V P Kandidov, O G Kosareva and H Schroeder, Can. J. Phys. 83, 863 (2005).

15 A. Couairon and A. Mysyrowicz, Phys. Rep. 441, 47 (2007).

${ }^{16}$ S. Tzortzakis, B. Prade, M. Franco, and A. Mysyrowicz, Opt. Commun. 181, 123 (2000).

${ }^{17}$ H. Pepin et al., Phys. Plasmas 8, 2532 (2001).

${ }^{18}$ M. Rodriguez et al., Opt. Lett. 27, 772 (2002).

${ }^{19}$ A. Houard, C. D’Amico, Y. Liu, Y. B. Andre, M. Franco, B. Prade, A. Mysyrowicz, E. Salmon, P. Pierlot, and L.-M. Cleon, App. Phys. Lett. 90, 171501 (2007).

${ }^{20}$ B. Forestier et al., AIP Advances 2, 012151 (2012).

${ }^{21}$ J. Kasparian et al., Science 301, 61 (2003).

${ }^{22}$ B. Forestier, A. Houard, M. Durand, Y. B. André, B. Prade, J.-Y. Dauvignac, F. Perret, Ch. Pichot, M. Pellet, and A. Mysyrowicz, Appl. Phys. Lett. 96, 141111 (2010).

${ }^{23}$ Y. Brelet, A. Houard, B. Forestier, Y. Liu, B. Prade, J. Carbonnel, Y.-B. André, L. Arantchouk, A. Mysyrowicz., Appl. Phys. Lett. 100, 181112 (2012).

${ }^{24}$ S. Tumanski, Meas. Sci. Technol. 18, (2007).

\section{Figures}

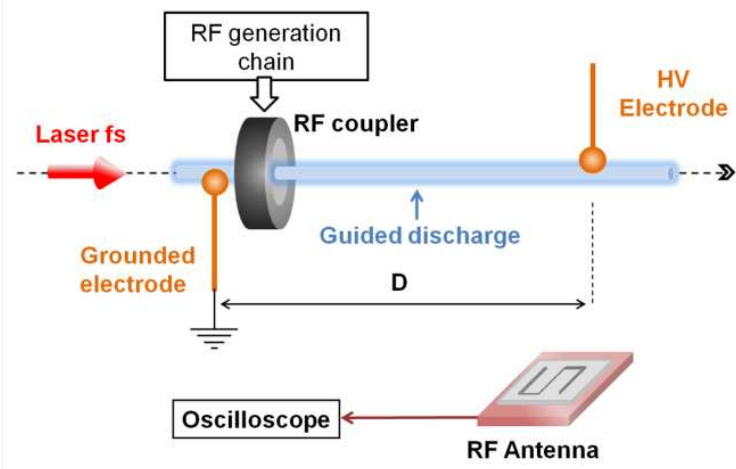

FIG. 1. (Color online) Experimental setup.

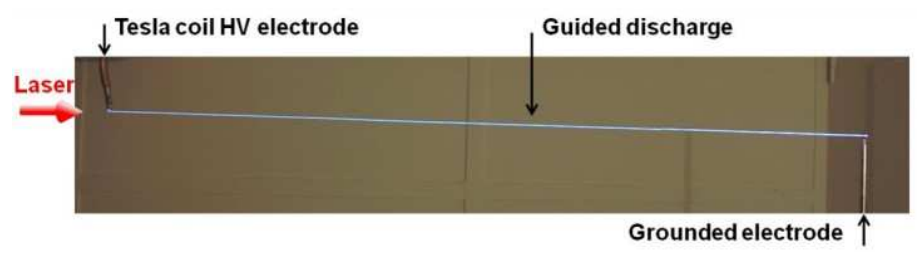

FIG. 2. Photography of a laser guided discharge for an interelectrode distance $\mathrm{D}=96 \mathrm{~cm}$. 


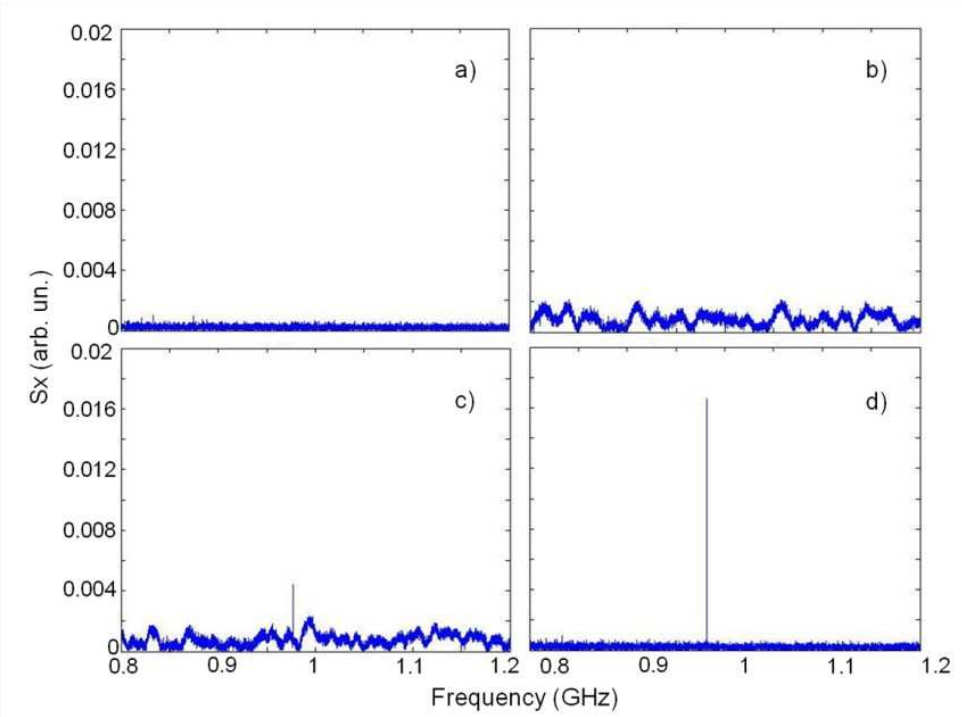

FIG. 3. Power spectral density $S_{x}$ for an injected frequency $F=970 \mathrm{MHz}$, for an interelectrode distance $\mathrm{D}=5 \mathrm{~cm}$ and a $\mathrm{DC}$ bias voltage $\mathrm{V} \approx 40 \mathrm{kV}$. a) Signal detected with RF source on but no discharge, b) signal in the presence of discharge but without RF, c) signal with discharge and RF, d) signal obtained when the discharge is replaced by a metallic copper rod.

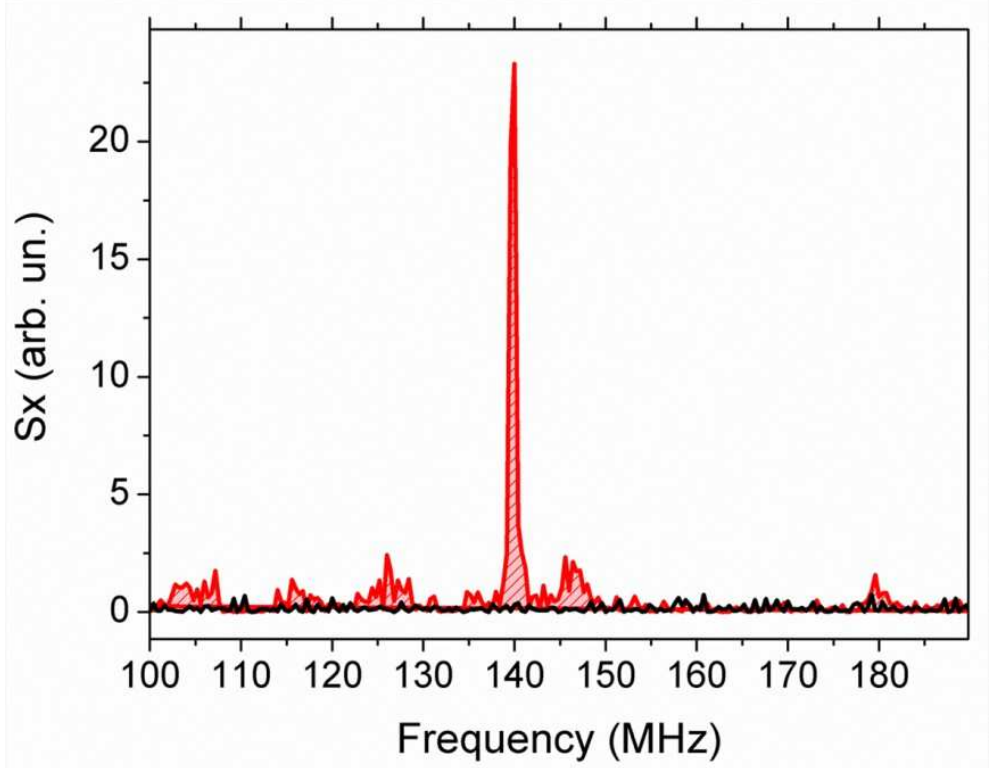

FIG. 4. Power spectral density $S_{x}$ for an injected frequency $F=140 \mathrm{MHz}$. Black curve is the ambient white-noise without guided discharge but with RF feeding in the solenoid coil at $140 \mathrm{MHz}$, showing perfect shielding. The guided discharge has a length $\mathrm{D}=96 \mathrm{~cm}$. 